\title{
La silicosis: ¿un problema de salud pública prioritario para Chile?
}

\author{
SYLVIA GALLEGUILLOS B.***, MARISOL CONCHA B.*,***, GUSTAVO CONTRERAS T.*,****, \\ GRETCHY ABARCA C.*,*****, ALBERTO MUÑOZ V.*,******, ESTEBAN VALENZUELA S.*,*******, \\ PABLO VILLAR M. $* * * * * * * * *$, MATÍAS LIBUY R. $* * * * * * * * * *$ y FRANCISCA CRISPI G. $* * * * * * * * * *$
}

\section{Silicosis: a relevant public health issue in Chile?}

Work related diseases and common diseases are covered by separated health systems in Chile. Chilean Ministry of Health focuses on common diseases, making work related diseases almost absent from public health policies. In this article current national and international information about the magnitude and impact of silicosis is reviewed. Although the quality of the national information is suboptimal, it is possible to estimate in several hundreds of thousands the number of workers exposed to silica dust, tens of thousands those under medical surveillance and thousands those currently affected by the disease. Albeit, additional efforts need to be made to estimate the burden of this disease on the Chilean population, information gathered in this article suggests that it is a relevant public health issue, deserving more importance among public policies in our country.

Key words: Silicosis, epidemiology, prevention and control, prevalence, mortality.

\section{Resumen}

Las enfermedades comunes y laborales son atendidas en distintos sistemas de aseguramiento de la salud en Chile. Las politicas públicas emanadas del Ministerio de Salud se enfocan en las enfermedades comunes, relegando a un rol secundario a aquellas ocasionadas por la actividad laboral de las personas. En este artículo, los autores revisan información actualizada tanto nacional como internacional con el fin de determinar si la silicosis constituye un problema de salud relevante. Aunque la calidad de la información nacional no es óptima, su revisión permite estimar que son cientos de miles los trabajadores expuestos a sílice cristalina, que hay varias decenas de miles bajo vigilancia médica y que son más de mil los portadores de la enfermedad. Si bien es necesario hacer mayores esfuerzos para estimar la carga que esta patología representa para la sociedad chilena, la información recopilada indica que constituye un problema de salud pública relevante que debiera tener mayor protagonismo en las políticas públicas de nuestro país.

Palabras clave: Silicosis, epidemiología, prevención, prevalencia, mortalidad.

Financiamiento: Proyecto FONIS SA13I20137.

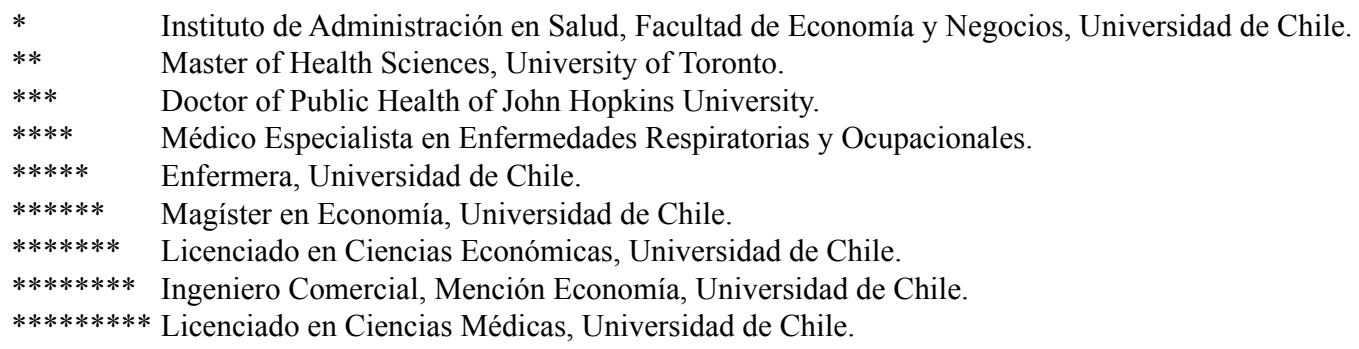




\section{Introducción}

La silicosis no es considerada un problema de salud pública prioritario en Chile. En la Estrategia Nacional de Salud para el Cumplimiento de los Objetivos Sanitarios de la Década 2011-2020 ${ }^{1}$, se plantean 8 objetivos estratégicos dentro de los cuales se menciona la necesidad de "disminuir la mortalidad por accidentes del trabajo" y "disminuir la incapacidad laboral por enfermedades profesionales". La inclusión de objetivos relacionados con los accidentes y las enfermedades profesionales en la estrategia se debe a la importancia que organismos como la Organización Internacional del Trabajo (OIT) confieren a estos temas. Por ejemplo, las estadísticas de la OIT indican que anualmente fallecen 185 millones de trabajadores y enferman otros 160 millones como resultado de la actividad productiva $^{1}$. El legislador reconoce que mejorar las condiciones de trabajo en Chile es importante para la salud de los trabajadores, para aumentar la productividad, para mejorar el crecimiento económico y los indicadores de desarrollo humano; sin embargo, también reconoce que uno de los problemas fundamentales es que se desconoce la magnitud del problema generado por este tipo de enfermedades en nuestro país ${ }^{2}$.

Reconociendo la importancia de las enfermedades profesionales, el legislador plantea como meta para la década, "disminuir un 30\% la tasa anual proyectada de licencias por incapacidad laboral por enfermedad profesional de causa musculo-esquelética de extremidad superior en población protegida por la Ley 16.744". Respecto de la silicosis reconoce la existencia de programas específicos, pero no plantea metas concretas, relegando a esta patología a un lugar secundario junto con el resto de las enfermedades profesionales ${ }^{i}$. Pudiera explicar esta decisión del legislador, el que tanto para las muertes por accidentes ${ }^{1}$ como para las patologías músculo-esqueléticas ${ }^{1}$, se dispone de datos que muestran la importancia de estos problemas de salud, en tanto para el resto de las patologías, entre las que se encuentra la silicosis, no hay información. La hipótesis de los autores es que aunque la información cuantitativa que existe para relevar la importancia de la sili-

En Chile en el año 2007, y por iniciativa de la OIT y la OMS, los ministerios de Salud y Trabajo y Previsión Social firmaron una declaración conjunta señalando el compromiso del gobierno de Chile de trabajar para lograr erradicar la silicosis al año 2030. A pesar de ello, la enfermedad no forma parte de las metas de salud para la década 2010-2020. cosis no es de buena calidad, es posible hacer esfuerzos para aproximarse a conocer la dimensión real del problema, incluso permitiendo alimentar modelos estadísticos y epidemiológicos que respalden el postulado de que la silicosis es un problema de salud pública que no debe ser relegado. Esto, ya que se sabe que la silicosis afecta a miles de trabajadores, generando morbilidad y mortalidad con importantes consecuencias sociales y económicas para el afectado y su familia. Siendo una enfermedad absolutamente prevenible, debiera ocupar un lugar de importancia en las políticas de salud pública de nuestro país.

En este artículo los autores analizan la información nacional e internacional respecto de la importancia de la silicosis como problema de salud pública.

\section{Método}

El método utilizado en este estudio consiste en estimar la prevalencia de la silicosis en base al análisis de datos sobre la población potencialmente expuesta, el registro de la cobertura de los programas de vigilancia ejecutados por las mutualidades de empleadores y del análisis de algunas estadísticas respecto de la enfermedad, utilizando información secundaria. Adicionalmente, estos resultados se contrastan con los obtenidos en una revisión de la literatura internacional.

\section{Trabajadores potencialmente expuestos}

La primera estimación del número de trabajadores potencialmente expuestos a sílice en Chile (laboran más del $30 \%$ de la jornada semanal en actividades que generan partículas de sílice cristalina en fracción respirable) corresponde al estudio realizado por Bernales y colaboradores en el Instituto de Salud Pública (ISP) y publicado en el año 2008,4. Dichos autores utilizaron información estadística correspondiente al año 2005. En el presente estudio se actualizaron las estimaciones al 2011, que es el último año para el que se dispone de información desagregada. Las fuentes utilizadas fueron la Encuesta Nacional Industrial Anual (ENIA 2011), el Anuario 2013 del Servicio Nacional de Geología y Minería (SERNAGEOMIN) el cual contiene datos históricos y las estadísticas nacionales del INE provenientes de la Encuesta Nacional de Empleo (ENE) del último trimestre de 2011. Tres son los sectores que presentan niveles de riesgo mayor: minas y canteras, construcción e industrias manufactureras. Para el primero, se extrajeron los datos del SERNAGEOMIN y del INE (respectivamente). Para el sector industrial, la actualización fue un 
tanto más compleja, ya que la ENIA 2011, a diferencia de la utilizada por los autores, presenta algunas categorías o variables de empleo de manera agregada ${ }^{\text {ii }}$.

Adicionalmente, se utilizó la información disponible en la Superintendencia de Seguridad Social, para estimar el número de trabajadores potencialmente expuestos en los Organismos Administradores de la Ley 16.744 (OAL) a saber, Asociación Chilena de Seguridad (ACHS), Mutual de la Cámara Chilena de la Construcción (CChC), Instituto de Seguridad del Trabajo (IST) e Instituto de Seguridad Laboral (ISL).

\section{Trabajadores expuestos y portadores de silicosis según los organismos administradores de la Ley 16.744}

A la información sobre trabajadores potencialmente expuestos a sílice se agrega aquella presentada por los OAL en agosto de 2014, en una actividad realizada como parte de las acciones del Plan Nacional para la Erradicación de la Silicosis (PLANESI) ${ }^{4}$. En ella las mutualidades de empleadores presentaron sus estadísticas sobre empresas, trabajadores expuestos y portadores de silicosis, de acuerdo a instrucciones emanadas de la Superintendencia de Seguridad Social ${ }^{5}$.

\section{Presencia de casos de silicosis en las salas ERA}

Otra fuente de información analizada corresponde al estudio sobre la prevalencia de la silicosis en las salas de atención de pacientes portadores de Enfermedades Respiratorias del Adulto (salas ERA), cuyos resultados preliminares fueron entregados al Ministerio de Salud en el año $2012^{7}$. En este estudio, focalizado en el personal de salud de las salas ERA de las regiones III, IV y Metropolitana, se aplicó un cuestionario para identificar pacientes con antecedentes de exposición a sílice, estimar el grado de exposición, antecedentes de evaluación en COMPIN (Comisión de Medicina Preventiva e Invalidez), así como estadígrafos demográficos.

\section{Silicosis en la literatura internacional}

Se realizó una búsqueda bibliográfica en la literatura internacional utilizando la siguiente estrategia:

ii En particular, para las empresas de menos de 50 trabajadores se incluyó el personal directivo y los propietarios y no se consideró el personal administrativo ni de servicios personales. Para las empresas de 50 a más trabajadores se excluyó las dos variables anteriormente mencionadas.
Criterios de selección de los artículos: Se incluyó todo tipo de estudios cuya población en estudio fueran trabajadores expuestos a sílice. Sólo se revisó artículos en idioma español e inglés, de 10 o menos años de antigüedad, que tuvieran el artículo disponible y que incluyeran las variables de interés de nuestro estudio.

Variable de respuesta: Se analizaron los artículos en cuanto a 2 variables: prevalencia y mortalidad.

Variable de exposición: Número de años de exposición a sílice.

Co-Variables: Se consideraron datos demográficos de los sujetos (edad y género), rubro de trabajo y criterios diagnósticos de silicosis.

Estrategia de búsqueda: Para la revisión de la literatura se realizó una búsqueda on-line en la Biblioteca Cochrane, MEDLINE (vía Pubmed) y Embase (DialogStar). Se utilizó las siguientes palabras clave: Silicosis prevalence, Silicosis incidence, Silicosis prevention, Silicosis cost effectiveness, Silicosis effectiveness, Pneumoconiosis prevention, Pneumoconiosis cost effectiveness y Pneumoconiosis effectiveness.

\section{Resultados}

Trabajadores potencialmente expuestos a sílice

En las Tablas 1 y 2 se presentan los resultados de la actualización de la estimación realizada por Bernales et al. En ellas se aprecia que el número de trabajadores potencialmente expuestos a sílice aumentó significativamente entre los años 2005 y 2011 desde 347.662 a 440.801 personas, variación explicada por el aumento de la Fuerza de Trabajo Ocupada (FTO).

Mientras el número de trabajadores en el rubro de la construcción no cambió significativamente, sí se aprecia una disminución importante en el rubro de industria y manufactura y un incremento sustancial en minas y canteras. El porcentaje de trabajadores potencialmente expuestos se mantiene en la construcción y aumenta en las otras dos ramas económicas.

La Tabla 2 presenta el número de trabajadores potencialmente expuestos a sílice según el Organismo Administrador de la Ley 16.744. Cabe destacar que la suma de todos los trabajadores en esa condición es sólo levemente inferior $(95,2 \%)$ a la calculada en la Tabla 1 . El número de trabajadores por rama de actividad económica es sustancialmente distinto, excepto para la construcción. Las estimaciones presentadas en la Tabla 1 indican que en la industria manufacturera hay 4.950 trabajadores potencialmente expuestos, 
Tabla 1. Trabajadores potencialmente expuestos a sílice por rama de actividad económica. Comparación 2005-2011

\begin{tabular}{|lcccccc|}
\hline Rama económica & $\mathbf{n}$ & $\begin{array}{c}\mathbf{2 0 0 5} \\
\mathbf{\% R E}\end{array}$ & $\mathbf{\% F T O}$ & $\mathbf{n}$ & $\begin{array}{c}\mathbf{2 0 1 1} \\
\mathbf{\% R E}\end{array}$ & \%FTO \\
\hline Industria y manufactura & 10.672 & 1,3 & 0,2 & 4.950 & 14,3 & 0,06 \\
\hline Minas y canteras & 33.619 & 39,0 & 0,5 & 98.596 & 50,0 & 1,22 \\
Construcción & 303.371 & 56,5 & 4,7 & 337.255 & 56,5 & 4,18 \\
Total & 347.662 & & 5,4 & 440.801 & & 5,46 \\
\hline
\end{tabular}

RE: Rama económica, FTO: Fuerza de trabajo ocupada. Fuente: Bernales et al. ${ }^{3}$ y elaboración propia.

Tabla 2. Número de trabajadores potencialmente expuestos a sílice según organismo administrador de la Ley 16.744. Año 2011

\begin{tabular}{|lrrrrc|}
\hline Rama económica & ACHS & CChC & IST & ISL & Total \\
Industria y manufactura & 30.103 & 14.180 & 6.876 & 5.400 & 56.559 \\
Minas y canteras & 16.076 & 14.923 & 2.916 & 12.594 & 46.509 \\
Construcción & 60.762 & 185.732 & 25.729 & 44.147 & 316.370 \\
Total & 106.941 & 214.835 & 35.521 & 62.141 & 419.438 \\
\hline
\end{tabular}

ACHS: Asociación Chilena de Seguridad, CChC: Cámara Chilena de la Construcción, IST: Instituto de Seguridad del Trabajo, ISL: Instituto de Seguridad Laboral. Fuente: Elaboración propia.

Tabla 3. Número trabajadores expuestos, en vigilancia y portadores de silicosis, según los organismos administradores de la Ley 16.744. Período 2011 a 2013

\begin{tabular}{|lccccc|}
\hline & ACHS & CCHC & IST & ISL & Total \\
Trabajadores expuestos & $9.592^{* *}$ & $7.190 * *$ & $13.293 * * * *$ & $122 * *$ & 30.197 \\
Trabajadores en vigilancia & $13.890^{* *}$ & $35.222^{* * *}$ & $2.763 * * * *$ & $6 * *$ & 51.881 \\
Trabajadores con silicosis & $145^{*}$ & $344 *$ & $11 *$ & $46^{*}$ & 646 \\
\hline
\end{tabular}

*al 31 de diciembre de 2011, **al 31 de diciembre de 2012, *** años 2009 a 2012, ****al 31 de diciembre de 2013. ACHS: Asociación Chilena de Seguridad, CChC: Cámara Chilena de la Construcción, IST: Instituto de Seguridad del Trabajo, ISL: Instituto de Seguridad Laboral. Fuente: Presentación Organismos Administradores de la Ley 16.744, agosto de 2014, PLANESI.

cifra que se aproxima a la estimada sólo para el ISL. Para minas y canteras, el total de personas presentada en la Tabla 2 es un $47 \%$ del estimado de la Tabla 1.

\section{Trabajadores expuestos y portadores de silicosis según los organismos administradores de la Ley 16.744}

Debido a que las estimaciones sobre trabajadores potencialmente expuestos debieron hacerse sobre la base de información del año 2011, la comparación con la información sobre trabajadores expuestos, en vigilancia y portadores de silicosis debiera corresponder al mismo año. Desafortunadamente, la información entregada por los Organismos Administradores de la Ley 16.744 es heterogénea. Se presentan dos Tablas con los datos aportados por las Mutuales de Seguridad. La Tabla 3 presenta la información más próxima al 2011 y la Tabla 4 aquella correspondiente al 2013, que es el primer año en el que se cuenta con información comparable.

La Tabla 3 muestra el número de trabajadores portadores de silicosis para el año 2011 para cada OAL. En ese año la suma total era de 646 enfermos. Para el número de trabajadores expuestos a sílice, se presenta la información al 31 de diciembre de 2012, salvo para el IST que reporta sólo desde el 2013. El número de trabajadores en vigilancia corresponde al dato registrado al 31 de diciembre de 2012 para la ACHS y el ISL. La CChC presenta un consolidado que incluye los años 2009 a 2012 y el IST reporta el dato de fines de 2013. 
Tabla 4. Número de trabajadores expuestos, en vigilancia y portadores de silicosis, según los organismos administradores de la Ley 16.744. Año 2013

\begin{tabular}{|lrrrrr|}
\hline & ACHS & CCHC & IST & ISL & Total \\
Trabajadores expuestos & 16.887 & 6.607 & 13.293 & 3 & 36.790 \\
Trabajadores en vigilancia & 29.868 & 13.345 & 2.763 & 51 & 46.027 \\
Trabajadores con silicosis & 509 & 224 & 6 & 283 & 1.022 \\
\hline
\end{tabular}

ACHS: Asociación Chilena de Seguridad, CChC: Cámara Chilena de la Construcción, IST: Instituto de Seguridad del Trabajo, ISL: Instituto de Seguridad Laboral. Fuente: Presentación Organismos Administradores de la Ley 16.744, agosto de 2014, PLANESI.

La Tabla 4 muestra la misma información esta vez para el año 2013 para todos los OAL, permitiendo así una comparación más adecuada. Al analizar las Tablas 3 y 4, se aprecia un aumento de un $21,8 \%$ en el número total de trabajadores expuestos, una reducción del 9,9\% del número de trabajadores en vigilancia y un $58,2 \%$ de aumento del número total de enfermos.

Llama la atención la diferencia entre el ISL y el resto de los OAL, ya que es la única que presenta valores que difieren del resto en órdenes de magnitud.

Tabla 5. Pacientes con diagnóstico de EPOC atendidos en Salas ERA, con antecedentes de exposición a sílice

\begin{tabular}{|lcrr|}
\hline Región & $\begin{array}{c}\text { Pacientes } \\
\text { encuestados }\end{array}$ & $\begin{array}{c}\text { Exposición } \\
\text { dudosa }\end{array}$ & $\begin{array}{c}\text { Expuestos a } \\
\text { Sílice }\end{array}$ \\
\hline Atacama & 128 & 2 & $115(89,8 \%)$ \\
\hline Coquimbo & 587 & 47 & $383(65,2 \%)$ \\
\hline Metropolitana & 1.242 & 135 & $388(31,2 \%)$ \\
\hline Totales & 1.957 & 184 & $886(45,3 \%)$ \\
\hline
\end{tabular}

EPOC: Enfermedad Pulmonar Obstructiva Crónica. Salas ERA: Salas para la atención de pacientes portadores de enfermedades respiratorias del adulto. Fuente: Solar et al. ${ }^{6}$

\section{Silicosis en las salas ERA}

Este estudio efectuó un total de 1.957 encuestas distribuidas por región según se presenta en la Tabla 5.

Llama la atención que los 115 pacientes identificados como expuestos a sílice durante su vida laboral, representen el $90 \%$ de todos los que se atienden en las salas ERA de la región de Atacama. Aunque en menor proporción, los porcentajes de pacientes portadores de EPOC atendidos en esas salas en las regiones de Coquimbo y Metropolitana, son también inesperadamente altos.

En este estudio se identificó dos grupos de pacientes dentro de aquellos con antecedente de exposición a sílice. Aquellos con sospecha de silicosis, son quienes presentan opacidades redondas en baja profusión en su radiografía de tórax. En estos pacientes el diagnóstico definitivo no ha sido efectuado y por ello están en esta categoría condicional. El segundo grupo son aquellos que cuentan con una resolución COMPIN que indica que son portadores de silicosis (Tabla 6).

Puesto que, según la Tabla 5, el antecedente exposición a sílice es más frecuente entre los pacientes de las salas ERA de la región de Atacama, es esperable que en dicha región se obtenga la mayor prevalencia de la enfermedad. Lo que sí

Tabla 6. Pacientes con diagnóstico de EPOC atendidos en salas ERA, con diagnóstico previo y con sospecha de silicosis

\begin{tabular}{|lcccc|}
\hline Región & $\begin{array}{c}\text { Pacientes } \\
\text { encuestados }\end{array}$ & $\begin{array}{c}\text { Pacientes con } \\
\text { sospecha de silicosis }\end{array}$ & $\begin{array}{c}\text { Pacientes con } \\
\text { diagnóstico de silicosis }\end{array}$ & $\begin{array}{c}\text { Prevalencia estimada } \\
\text { de silicosis (\%) }\end{array}$ \\
\hline Atacama & 128 & 38 & 75 & $58,6(88,3)$ \\
Coquimbo & 587 & 69 & 155 & $26,4(38,2)$ \\
Metropolitana & 1.242 & 24 & 43 & $3,4(5,4)$ \\
Totales & 1.957 & 131 & 273 & $13,9(20,6)$ \\
\hline
\end{tabular}

"La prevalencia estimada de silicosis presenta dos valores: el primero es la razón entre los casos con silicosis reconocida por COMPIN y el segundo (entre paréntesis) la razón que se obtendría si todos los casos sospechosos fuesen reconocidos como enfermos profesionales. EPOC: Enfermedad Pulmonar Obstructiva Crónica. Salas ERA: Salas para la atención de pacientes portadores de enfermedades respiratorias del adulto. Fuente: Solar et al. ${ }^{6}$ 
es llamativo es que en esa región la tasa supere el $50 \%$. En promedio, una prevalencia de $14 \%$ (antes de incorporar los casos con sospecha de silicosis) resulta también muy alta.

\section{Silicosis en la literatura internacional}

La prevalencia de silicosis en la literatura internacional varía según rubro y método de diagnóstico utilizado (Tabla 7). En su mayoría corresponden a estudios de cohorte y transversales, llevados a cabo en un rubro determinado. El mayor número de estudios ocurren en los rubros de minería y construcción, se concentran en 5 países y en la mayoría el diagnóstico se basó en la radiografía de tórax, utilizando la clasificación OIT. Una excepción son los estudios de China, en los cuales se utilizó el "Chinese Silicosis Roentgen diagnostic criteria", criterio que tiene una concordancia de un $85 \%$ con la clasificación OIT.

En el rubro de la minería, la prevalencia oscila entre 3,2 y $36 \%$. Entre los estudios analizados, destacan dos con un tamaño de muestra elevado realizados por Chenel $2006^{8}$ y $2012^{9}$ en China. En el primero la prevalencia fue de $20 \%$ y en el segundo de 22,3\%. En ambos estudios se seleccionó una cohorte de mineros expuestos a sílice los que fueron vigilados por 22 y 33 años con radiografía de tórax anual y cada 3 años, respectivamente. En esta misma línea, Laney et al. ${ }^{10}$, en los Estados Unidos, estimó la prevalencia de silicosis en un $3,2 \%$ en una cohorte de 90.973 trabajadores de minas de carbón seguidos entre 1980 y 2008; el diagnóstico se realizó de acuerdo a la clasificación radiológica OIT con profusión de opacidades en grado 1 o mayor.
En el rubro de la construcción, Chinh V et al. ${ }^{11}$, en un estudio de 158 trabajadores de fábricas de ladrillos, reportó una prevalencia de silicosis de $10 \%$, diagnosticada a través de radiografías de tórax. Fan Y et al. ${ }^{12}$, reportaron una prevalencia de $9,34 \%$ en 3.417 trabajadores que construyeron túneles por espacio de 5 años. Otros trabajos comunican prevalencias similares en este rubro ${ }^{13}$.

En otros rubros, Murgia $\mathrm{N}$ et al. ${ }^{14}$, estimaron en $23 \%$ la prevalencia de silicosis en joyeros expuestos en promedio por 13,3 años. Oliver et al. ${ }^{15}$, no informaron casos de silicosis en 343 trabajadores de carreteras expuestos a polvo de sílice en promedio por 21,8 meses.

Respecto de mortalidad atribuible a silicosis, Bang et al. ${ }^{16}$, estimaron una tasa de mortalidad de 2,4 casos por millón de habitantes para 1981 y de 0,7 casos por millón en 2004 en los Estados Unidos. Esta cifra es concordante con la reportada por Mazurek et al., de 2,46 casos por millón de habitantes desde 1968 al 2004 ${ }^{17}$. En China, Chen $\mathrm{W}$ et $\mathrm{al} .{ }^{8}$, en un estudio de cohorte de 74.040 trabajadores de la minería y de fábricas de cerámica seguidos entre 1960-1974 y el 2003, estimó en $4,2 \%$ el porcentaje de muertes atribuibles a la exposición a sílice. En otro estudio del mismo primer autor sobre una cohorte de 7.837 trabajadores, la mortalidad alcanzó a un 7,04\% ${ }^{9}$.

En relación a las causas originarias de muerte, el estudio de Marinaccio et al., en una cohorte de 14.929 trabajadores portadores de silicosis, evidenció un aumento del riesgo de muerte al comparar con la población general de 1,1 $(95 \%$ IC: 1,03-1,18) para cáncer de pulmón, tráquea y bronquios, 1,78 (95\% IC: 1,42-2,23) para enfer-

Tabla 7. Estudios internacionales sobre prevalencia de silicosis en distintos grupos de trabajadores

\begin{tabular}{|c|c|c|c|c|c|c|}
\hline Autores & Rubro & Tipo de estudio & Diagnóstico & Prevalencia (\%) & $\mathbf{n}$ & País \\
\hline Murray et $\mathrm{al}^{20}$ & Minería & A Tendencia & Autopsia & $9,3-12,8$ & 16.454 & Sudáfrica \\
\hline Steen et $\mathrm{al}^{21}$ & Minería & Est transversal & Rx de Tórax & $26,6-31$ & 304 & Sudáfrica \\
\hline Trapido et $\mathrm{al}^{22}$ & Minería & Est transversal & Rx de Tórax & $22,0-36,0$ & 238 & Sudáfrica \\
\hline Meel et $\mathrm{al}^{23}$ & Minería & Est transversal & Rx de Tórax & 24,0 & 300 & Sudáfrica \\
\hline Churchyard $^{24}$ & Minería & Est transversal & Rx de Tórax & $18,3-19,9$ & 520 & Sudáfrica \\
\hline Girdler-Brawn ${ }^{25}$ & Minería & Est transversal & Rx de Tórax & 24,6 & 624 & Sudáfrica \\
\hline Park et $\mathrm{al}^{26}$ & Minería & Cohorte & Rx de Tórax & 27,0 & 779 & Sudáfrica \\
\hline Chen $2006^{8}$ & Minería & Cohorte & Rx de Tórax & 20,0 & 7.837 & China \\
\hline Chen $2012^{9}$ & Minería & Cohorte & Rx de Tórax & 22,2 & 74.040 & China \\
\hline Laney $^{10}$ & Minería & Est transversal & Rx de Tórax & 3,2 & 90.973 & USA \\
\hline Chinh $^{11}$ & Const. & Est transversal & Rx de Tórax & 10,0 & 158 & Vietnam \\
\hline Murgia $^{14}$ & Joyería & Est transversal & TAC de Tórax & 23,0 & 100 & Italia \\
\hline
\end{tabular}

Fuente: Elaboración propia. 
Tabla 8. Estudios internacionales sobre mortalidad atribuible a silicosis

\begin{tabular}{|lclcc|}
\hline Autores & $\mathbf{n}$ & Tipo de estudio & Mortalidad atribuible & País \\
\hline Chen $^{8}$ & 74.040 & Cohorte & $4,2^{\mathrm{a}}$ & China \\
${\text { Chen } \mathrm{W}^{9}}^{9}$ & 7.837 & Cohorte & $7,04^{\mathrm{a}}$ & China \\
Bang $^{16}$ & 6.326 & Cohorte & $0,7 / 1.000 .000^{\mathrm{b}}$ & USA \\
Mazurek $^{17}$ & & Caso control & $2,46 / 1.000 .000^{\mathrm{b}}$ & USA \\
\hline
\end{tabular}

${ }^{\mathrm{a}}$ Mortalidad atribuible a exposición de polvo de sílice. ${ }^{\mathrm{b}}$ Tasa de Mortalidad ajustada por millón de habitantes.

medades infecciosas, 2,15 (95\% IC: $1,84-2,51)$ para cáncer hepático y 2,89 (95\% IC: 2,20-3,8) para tuberculosis ${ }^{18}$.

\section{Comentarios}

Lo más llamativo de la información presentada en este análisis lo constituye la evidente disparidad de cifras existente entre las distintas fuentes consultadas. La estimación realizada en este estudio de un total de 440.801 trabajadores potencialmente expuestos para el año 2011, contrasta con la cifra de 419.438 obtenida al analizar la información de los Organismos Administradores de la Ley 16.744, puesto que se sabe que las mutualidades dan cobertura a no más del $70 \%$ de la FTO. Sin embargo, la relación entre ambas cifras (la segunda es un 95,2\% de la primera) puede ser real ya que se refiere a tres rubros donde pudiera ocurrir que la FTO con contrato sea cercana al $100 \%$. Esta presunción requiere de mayor investigación.

Nuestro intento por establecer una comparación entre las estimaciones de trabajadores potencialmente expuestos con los registros provenientes de los programas de vigilancia de los Organismos Administradores de la Ley, no pudo concretarse, ya que no se dispuso de la información requerida para el año 2011. La comparación más cercana posible de establecer es con el año 2013, e indica que los trabajadores expuestos y en vigilancia médica representan menos del $10 \%$ de los estimados potenciales, y que en conjunto reconocen un poco más de 1.000 trabajadores afectados. La presunción de que este último número está subestimado no sólo deriva de la reducida cobertura de los programas de vigilancia, sino del hecho de que al menos el $14 \%$ de los pacientes portadores de silicosis están siendo atendidos en las salas ERA bajo el diagnóstico de EPOC, no obstante ser en realidad enfermos portadores de silicosis. En una muestra representativa de 1.957 de estos pacientes, 273 de ellos corresponden a casos ya diagnosticados con la enfermedad. Esta cifra representa un incremento de un $26,7 \%$ en la reportada por los Organismos Administradores de la Ley.

Por otra parte, aun cuando se podría establecer una relación entre el número de enfermos y trabajadores en vigilancia con el objeto de estimar la prevalencia $(1.022 / 46.027=2,2 \%$, o $1.295 / 46.027=2,8 \%$ ), desafortunadamente son datos que no están relacionados. El ejemplo más claro surge de los datos del ISL el que, a pesar de tener a un reducido número de trabajadores en vigilancia, muestra una de las cifras más altas de enfermos. La explicación más plausible es que la mayor parte de los enfermos que registra esa institución provienen de consulta espontánea de los trabajadores a las COMPIN del país.

Existen a lo menos otras dos consideraciones importantes a tener en cuenta al intentar establecer la prevalencia de la enfermedad. La primera es que la silicosis es una patología que en general tiene un largo período de latencia, por lo que los pacientes que están siendo diagnosticados hoy son producto de una exposición de al menos 15 años de antigüedad. Un segundo elemento es que el riesgo de silicosis no termina al finalizar la vida laboral, por lo que es frecuente que el diagnóstico se haga en personas ya jubiladas. No es adecuado, por tanto, relacionar el número de expuestos o enfermos con la FTO, sino que debiera considerarse a todos aquellos que han estado alguna vez expuestos a dosis suficiente como para causar silicosis y aún se encuentran con vida. Al incorporar a la población mayor de 65 años a las estimaciones de personas potencialmente expuestas a sílice, la prevalencia puede aumentar significativamente.

Estas son algunas de las dificultades metodológicas que enfrenta la tarea de estimar la prevalencia de la silicosis. Tal vez esto explique que países como los Estados Unidos de Norteamérica ${ }^{20}$ puedan señalar cual es la morbilidad, la mortalidad por silicosis, por cáncer pulmonar derivada de la exposición a sílice, por falla renal 
o por otras patologías asociadas a esta exposición laboral, pero no pueden precisar cuál es la prevalencia de la enfermedad. Por otra parte, la revisión de la literatura reporta magnitudes muy variadas. La Tabla 7 presenta prevalencias que oscilan entre 1 y $36 \%$. Dicha variabilidad persiste aún en aquellos estudios que involucran varios miles de trabajadores (3,2 y $22 \%$ ). Este no es el caso respecto de los estudios sobre mortalidad por silicosis, los cuales presentan un rango de variación reducido que oscila entre 2,4 y 4,3\%.

Con todo, la información disponible en Chile $y$ que ha sido analizada en este estudio, permite asegurar que son cientos de miles los trabajadores potencialmente expuestos a sílice, que son decenas de miles los que se encuentran en programas de vigilancia de la salud por parte de los Organismos Administradores de la Ley y que son miles los que presentan la enfermedad. Si bien se requieren más estudios para estimar cual es la carga de enfermedad que la silicosis representa para nuestro país, con lo información disponible es posible asegurar que esta patología constituye un problema de salud pública que merece mayor atención por parte de los organismos del estado correspondientes.

\section{Agradecimientos}

Los autores agradecen al Sr. Juan Alcaíno L., quién aportó valiosa información sobre el procedimiento de estimación de trabajadores potencialmente expuestos a sílice utilizados en el estudio del Instituto de Salud Pública de Chile.

\section{Referencias}

1.- MINISTERIO DE SALUD. Estrategia Nacional de Salud para el Cumplimiento de los Objetivos Sanitarios de la Década 2011-2020. Santiago: Ministerio de Salud de la República de Chile; 2010. p. 173-9.

2. CONCHA M, LABBÉ J. Enfermedades profesionales: Una aproximación a su frecuencia. Ciencia \& Trabajo. 2007; 9: 117-20.

3. BERNALES B, ALCAÍNO J, SOLÍS R. Situación de Exposición Laboral a Sílice en Chile. Ciencia \& Trabajo 2008; 10: 1-6.

4. ALCAÍNO J, BERNALES B, SOLÍS R. Metodología para estimar el número de Trabajadores Potencialmente expuestos a Sílice en Chile. Documento de trabajo, Instituto de Salud Pública, 2007.

5. MINISTERIO DE SALUD Y MINISTERIO DEL TRABAJO Y PREVISIÓN SOCIAL. Plan Nacional para la erradicación de la Silicosis. Santiago: Ministerio de
Salud y Ministerio del Trabajo y Previsión Social de la República de Chile; 2008.

6. MINISTERIO DEL TRABAJO. Superintendencia de Seguridad Social. Circular 2971. Plan de Prevención de Accidentes del Trabajo y Enfermedades Profesionales 2014. Imparte Instrucciones a los Organismos Administradores de la Ley 16.744. Santiago: Ministerio del Trabajo República de Chile; 30 de diciembre de 2013.

7. SOLAR O, BERNALES P, GONZÁLEZ M J. Estudio transversal para la determinación de prevalencia de silicosis. Santiago: Universidad del Desarrollo; 2012. LE11: 757-273.

8. CHEN W, YANG J, CHEN J, BRUCH J. Exposures to silica mixed dust and cohort mortality study in tin mines: exposure-response analysis and risk assessment of lung cancer. Am J Ind Med 2006; 49: 67-76.

9. CHEN W, LIU Y, WANG H, HNIZDO E, SUN Y, SU $\mathrm{L}$, et al. Long-term exposure to silica dust and risk of total and cause-specific mortality in Chinese workers: a cohort study. PLoS Med 2012; 9: e1001206.

10. LANEY A S, PETSONK E L, ATTFIELD M D. Pneumoconiosis among underground bituminous coal miners in the United States: is silicosis becoming more frequent? Occup Environ Med 2010; 67: 652-6.

11.- CHINH V C, CHAI S K, HAI D N, TAKARO T, CHECKOWAY H, KEIFER M, et al. Pneumoconiosis among workers in a Vietnamese refractory brick facility. Am J Ind Med 2002; 42: 397-402.

12. FAN Y. Prevalence of silicosis among XiangyangChongqing Railway construction workers 40 years after dust exposure. Zhonghua Lao Dong Wei Sheng Zhi Ye Bing ZaZhi 2014; 32: 528-9.

13. VALIANTE D J, SCHILL D P, ROSENMAN K D, SOCIE E. Highway repair: a new silicosis threat. Am J Public Health 2004; 94: 876-80.

14. MURGIA N, MUZI G, DELL'OMO M, SALLESE D, CICCOTOSTO C, ROSSI M, et al. An old threat in a new setting: High prevalence of silicosis among jewelry workers. Am J Ind Med 2007; 50: 577-83.

15.- OLIVER L C, MIRACLE-MCMAHILL H. Airway disease in highway and tunnel construction workers exposed to silica. Am J IndMed 2006; 49: 983-96.

16.- BANG K M, ATTFIELD M D, WOOD J M, SYAMLAL G. National trends in silicosis mortality in the United States, 1981-2004. Am J Ind Med 2008; 51: 633-9.

17.- MAZUREK J M, ATTFIELD M D. Silicosis mortality among young adults in the United States, 1968-2004. Am J Ind Med 2008; 51: 568-78.

18.- MARINACCIO A, SCARSELLI A, GORINI G, CHELLINI E, MASTRANTONIO M, UCCELLI R, et al. Retrospective mortality cohort study of Italian workers compensated for silicosis. Occup Environ Med 2006; 63: 762-5.

19.- DEPARTMENT OF LABOR. Occupational Safety and Health Administration. 29 CFR Parts 1910, 1915, and 1926. 2013; Occupational Exposure to Respirable Crys- 
talline Silica. Proposed Rule.

20.- MURRAY J, KIELKOWSKI D, REID P. Occupational disease trends in black South African gold miners. An autopsy-based study. Am J Respir Crit Care Med 1996; 153: 706-10.

21.- STEEN T W, GYI K M, WHITE N W, GABOSIANELWE T, LUDICK S, MAZONDE G N, et al. Prevalence of occupational lung disease among Botswana men formerly employed in the South African mining industry. Occup Environ Med 1997; 54: 19-26.

22.- TRAPIDO A S, MQOQI N P, WILLIAMS B G, WHITE N W, SOLOMON A, GOODE R H, et al. Prevalence of occupational lung disease in a random sample of former mineworkers, Libode District, Eastern Cape Province, South Africa. Am J Ind Med 1998; 34: 305-13.

23. MEEL B L. Patterns of lung diseases in former mine workers of the former Republic of the Transkei: an X-ray-based study. Int J Occup Environ Health. 2002;
8: $105-10$

24.- CHURCHYARD G J, EHRLICH R, TEWATERNAUDE J M, PEMBA L, DEKKER K, VERMEIJS M, et al. Silicosis prevalence and exposure-response relations in South African goldminers. Occup Environ Med 2004; 61: 811-6.

25.- GIRDLER-BROWN B V, WHITE N W, EHRLICH R I, CHURCHYARD G J. The burden of silicosis, pulmonary tuberculosis and COPD among former Basotho goldminers. Am J Ind Med 2008; 51: 640-7.

26.- PARK H H, GIRDLER-BROWN B V, CHURCHYARD G J, WHITE N W, EHRLICH R I. Incidence of tuberculosis and HIV and progression of silicosis and lung function impairment among former Basotho gold miners. Am J Ind Med 2009; 52: 901-8.

27.- ZHANG M, ZHENG Y D, DU X Y, LU Y, LI W J, QI C, et al. Silicosis in automobile foundry workers: a 29-year cohort study. Biomed Environ Sci 2010; 23: 121-9.
Correspondencia a:

Ing. Sylvia Galleguillos, MHSc

Instituto de Administración en Salud

Facultad de Economía y Negocios

Universidad de Chile

Dirección: Diagonal Paraguay \#257, Torre 26, Of

2204-Santiago

Email: sgallegu@fen.uchile.cl 